\title{
Partial to Full Image Registration Based on Candidate Positions and Multiple Correspondences*
}

\author{
Carlos Francisco Moreno-García, Xavier Cortés, and Francesc Serratosa \\ Universitat Rovira i Virgili, \\ Departament d'Enginyeria Informàtica i Matemàtiques, Spain \\ carlosfrancisco.moreno@estudiants.urv.cat, \\ \{xavier.cortes, francesc.serratosa\}@urv.cat
}

\begin{abstract}
In some image-registration based applications, it is more usual to detect a low quality and tiny partial image rather than a full sample (forensic palmprint recognition, satellite images, object detection in outdoor scenes ...). In these cases, the usual registration methods fail due to the great amount of outliers that have to be detected while comparing a tiny image (object to be registered) to a full image (object in the database). In this paper, we present an image registration method that explicitly considers a great amount of outliers. In a first step, the method selects some candidate points to be the centres of the partial image. In a second step, these candidates are refined until selecting one through a multiple correspondence method. Experimental validation shows that the algorithm can outperform state of the art identification methods given the image to be identified a tiny and partial sample.
\end{abstract}

Keywords: Sub-Image Registration, Hough Method, Candidate Voting, Hungarian Algorithm, ICP.

\section{$1 \quad$ Introduction and Related Work}

Image registration in computer vision tries to determine which parts of one image correspond to which parts of another image. This problem often arises at the early stages of many computer vision applications such as scene reconstruction, object recognition and tracking, pose recovery and image retrieval. Interesting image registration surveys are [1] and [2], which explain the problematic of this goal. It is of basic importance to develop effective methods that are both robust in two aspects a) Being able to deal with noisy measurements and b) Having a wide field of application.

The two typical steps involved in the solution of the image registration problem are the following [3]. First, some salient points are selected from both images [4] and then a set of tentative matches between these sets of points is computed [5], [6].

\footnotetext{
* This research is supported by the CICYT project DPI2013-42458-P, by project TIN201347245-C2-2-R and by Consejo Nacional de Ciencia y Tecnologías (CONACyT Mexico).
} 
Second, these tentative matches can be further refined by a process of outlier rejection [7] that eliminates the spurious correspondences or alternatively, they can be used as starting point of some optimization scheme to find a different and more consistent set [8]. Recently, some other methods have appeared which take into consideration salient points grouped in several sets of points, since they assume different transformations are applied to each point set [9].Moreover, some methods have been presented which register several images at a time [10], [11] to increase the probability of finding successful matches.

The main drawback of all of these methods is that their ability to obtain a dense correspondence set strongly depends on the reliability of the tentative correspondences. In some image-registration based applications (forensic palmprint recognition, satellite images ...), it is more usual to detect a tiny partial image rather than a full sample. In these cases, the tentative initial correspondences returned by the first step fail due to the great amount of outliers that have to be detected while comparing a tiny image to a full image. Thus, the second step (usually highly dependent on these initial correspondences) is not able to recover neither the correct correspondences nor the transformation matrix from the tiny image to the large image.

In this paper, we present an image registration method that explicitly considers that one of the images is a noisy and small part of the other one. In section 2, we describe the method. In section 3, we give a brief explanation about the palmprint identification state of the art, as well as an explanation of how we have applied our method to a partial palmprint registration case. Finally, we conclude the paper in section 4.

\section{Partial to Full Image Registration}

Consider we want to align a small image to a large image. We suppose the small one shows part of the larger one. For this reason, we say the small image is a partial image $P$ and the larger one is a full image $F$. Both images are represented by their salient points, $\left(x^{P}, y^{P}\right)=\left\{\left(x_{1}^{P}, y_{1}^{P}\right), \ldots,\left(x_{|P|}^{P}, y_{|P|}^{P}\right)\right\}$ and $\left(x^{F}, y^{F}\right)=$ $\left\{\left(x_{1}^{F}, y_{1}^{F}\right), \ldots,\left(x_{|F|}^{F}, y_{|F|}^{F}\right)\right\}$ together with their features $f^{P}=\left\{f_{1}^{P}, \ldots, f_{|P|}^{P}\right\}$ and $f^{F}=\left\{f_{1}^{F}, \ldots, f_{|F|}^{F}\right\}$. The number of salient points is $|P|$ and $|F|$, respectively.

In the first step, $k$ positions $\left(x_{1}^{c}, y_{1}^{c}\right), \ldots,\left(x_{k}^{c}, y_{k}^{c}\right)$ on the full image $F$ are selected as candidates to be the centre of the partial image $P$ if both images were aligned. Then, the full image $F$ is split in sub-images $F_{1}, \ldots, F_{k}$, in which the centre of each image $F_{a}$ is the candidate position $\left(x_{a}^{c}, y_{a}^{c}\right)$. Each split image $F_{a}$ is represented by their set of salient points $\left(x^{F_{a}}, y^{F_{a}}\right)=\left\{\left(x_{1}^{F_{a}}, y_{1}^{F_{a}}\right), \ldots,\left(x_{\left|F_{a}\right|}^{F_{a}}, y_{\left|F_{a}\right|}^{F_{a}}\right)\right\}$ and also their corresponding set of features $f^{F_{a}}=\left\{f_{1}^{F_{a}}, \ldots, f_{\left|F_{a}\right|}^{F_{a}}\right\}$. Note that the number of extracted salient points in the partial image $|P|$ and the split ones $\left|F_{a}\right|$ can be different. Moreover, $|F| \leq \sum_{a=1}^{k}\left|F_{a}\right|$, since the split images can overlap. 




Fig. 1. Diagram of the Registration method

In the second step, the algorithm seeks the best alignment between the salient points $\left(x^{P}, y^{P}\right)$ of the partial image $P$ and the salient points $\left(x^{F_{a}}, y^{F_{a}}\right)$ of each of the split images $F_{1}, \ldots, F_{k}$. To obtain these alignments, not only the salient point positions are used but also their extracted features, more precisely, features $f^{P}$ and $f^{F a}$. Thus, $k$ distances $D_{1}, \ldots, D_{k}$ and $k$ alignments (also called homographies) $H_{1}, \ldots, H_{k}$ are computed. Finally, the method selects the image that obtains the minimum distance and returns the alignment $H_{P, F}$ between $P$ and $F$ that obtains this distance. On the following subsections we will explain in a deeper form each of the two steps of our PF-Registration method.

\subsection{Selecting Some Position Candidates}

Figure 2 shows the main structure of the first step of our method. It is based on a Generalized Hough Transform [12], [13], [14]. As commented in the previous section, the method first obtains $|P|$ and $|F|$ salient points (position and features) of both images.

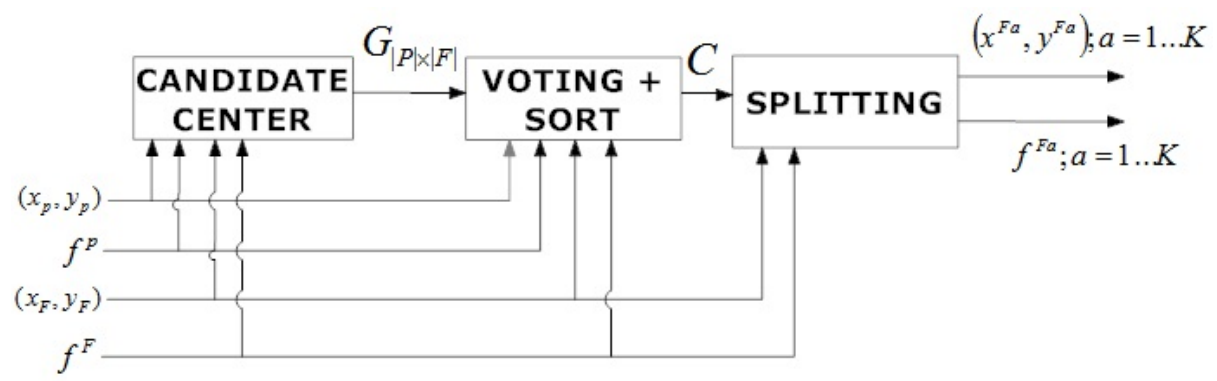

Fig. 2. Diagram of Step 1

We define a $|P| x|F|$ matrix G[i,j]. The Candidate Centre module fills each cell of $\mathrm{G}[\mathrm{i}, \mathrm{j}]$ with the position $\left(x_{i j}^{C}, y_{i j}^{C}\right)$ on the full image $F$ that the centre of the partial image $(\bar{x}, \bar{y})$ would obtain if the point $\left(x_{i}^{P}, y_{i}^{P}\right)$ on the partial image where mapped to 
the point $\left(x_{j}^{F}, y_{j}^{F}\right)$ on the full image. There are several forms to obtain these centres [14]. They can use only one or several points, and also some information extracted from the features, such us angle information. The aim of this process is to detect the spatial relations on both images. If $s$ points in $P$ and $s$ points in $F$ have the same relative position, then there is going to be $s$ cells of $\mathrm{G}[\mathrm{i}, \mathrm{j}]$ with the same value. These cells are the ones that the mapping between points on both images is the correct one.

When matrix $\mathrm{G}$ is filled, then the Voting and Sort module generates an ordered list $C$ of the positions $\left(x_{i j}^{C}, y_{i j}^{C}\right)$ found inG, where $C=\left\{\left(x_{1}^{c}, y_{1}^{c}\right), \ldots,\left(x_{T}^{c}, y_{T}^{c}\right)\right\}$ through a clustering and voting process. List $C$ is set in a descendent order. That is, the positions with the most votes are the first ones. Note that $T \leq|P| x|F|$. The voting process counts the number of centres grouped by the clustering process and also that their features are considered to be similar enough. The clustering process considers two centre points $\left(x_{i j}^{C}, y_{i j}^{C}\right)$ and $\left(x_{i, j}^{C}, y_{i, j}^{C}\right)$ have to be the same if they are close enough. That is, the distance is lower than a spatial threshold, defined bydist $_{P, M}^{\text {position }}\left(\left(x_{i j}^{C}, y_{i j}^{C}\right),\left(x_{i, j}^{C}, y_{i, j}^{C}\right)\right)<T_{s}$. Thus, the Voting and Sort module counts and orders the cells in $\mathrm{G}$ such that $\operatorname{dist}_{P, M}^{\text {position }}\left(\left(x_{i j}^{C}, y_{i j}^{C}\right),\left(x_{i, j^{\prime},}^{C} y_{i, j}^{C}\right)\right)<T_{s}$ and $\operatorname{dist}_{P, M}^{\text {feature }}\left(f_{i}^{F}, f_{j}^{P}\right)<T_{f}$. Note that both distances areparameterised. In the case of dist $_{P, M}^{\text {position }}$, this is done to be independent of the rotation and scale. In the case of $d_{i s t} t_{P, M}^{\text {feature }}$, this is done to be independent of some global feature distortions.

Finally, with the best $K$ candidates to be the centre of the partial image on the full image, the set of points $\left(x^{F}, y^{F}\right)$ and the set of features $f^{F}$ are split in $K$ point sets $\left(x^{F_{a}}, y^{F_{a}}\right), 1 \leq a \leq K$ and $K$ feature sets $f^{F_{a}}, 1 \leq a \leq K$. Each point in $\left(x_{i}^{F}, y_{i}^{F}\right)$ is included in the set $F_{a}$ if $\operatorname{dist}_{P, M}^{\text {position }}\left(\left(x_{i}^{F}, y_{i}^{F}\right),\left(x_{a}^{C}, y_{a}^{C}\right)\right) \leq T_{r}$. The threshold $T_{r}$ represents the maximum radius of the set, meaning the maximum distance between any point and the centre of the set. Usually, it is determined depending on the radius of the partial set $\left(x^{P}, y^{P}\right)$. The set $f^{F_{a}}$ is defined congruent with the set $\left(x^{F_{a}}, y^{F_{a}}\right)$.Parameter $K$ is application dependent, but it is commonly set as a value equal or lower than 4 to avoid spurious candidate centres.

\subsection{Best Candidate Selection through Multiple Correspondences}

Figure 3 shows the second step of our registration method.

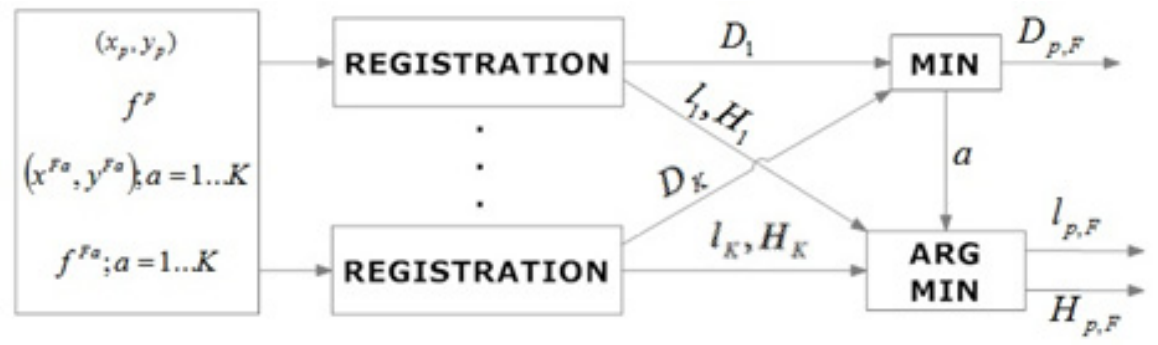

Fig. 3. Diagram of Step 2 
In this second step, the method first seeks the distances $D_{a}=\operatorname{dist}\left(P, F_{a}\right) ; 1 \leq a \leq$ $K$ and the correspondences $l_{a}$ between the points in each set, and also the homographies $H_{a}$ that transform $P$ to $F_{a}$. Several algorithms can be used to find these correspondences and/or homographies. These algorithms use the positional information $\left(x^{F_{a}}, y^{F_{a}}\right)$ and $\left(x^{P}, y^{P}\right)$ and also their features $f^{F_{a}}$ and $f^{P}$. For example, the Hungarian method [15] or ICP [5] (when no outliers are considered), the RANSAC method [7] that considers the presence of outliers, the Fast Bipartite Graph Matching [30]that considers second order information and more sophisticated ones [8]. Even a greedy algorithm that simply selects the best option without considering the other candidates could be used.

We wish to select the set of points $\left(x^{F_{a}}, y^{F_{a}}\right)$ that obtained the minimum distance $D_{a}$. This is because we assume $D_{a}$ is agood enough approximation ofdist $(P, F)$. Moreover, we also assume the correspondence an alignment (homography) between $P$ and $F$ approximates the correspondence $l_{a}$ and homography $H_{a}$. Therefore, $l_{P, F}=l_{a}$ and $H_{P, F}=H_{a}$.

Breaking down the full image in a set of candidates has two advantages. On the one hand, the computational cost of obtaining the $K$ distances $D_{a}$ is lower than obtaining directly the value $D_{P, F}$. On the other hand, the sub-optimal algorithm tends to obtain a more precise local minimum.

\section{Practical Validation}

Several methods have been presented for palmprint recognition [17], [18]. Along many other studies, an initial approach modelled by Zhang \& Kong [19] proposes a low resolution image matching based on the palmprint ridges. Funada et al. [20] create a novel algorithm for minutiae matching using crease elimination. Nevertheless, these models cannot be applied while performing partial to full palmprint matching. Derivate from such approach, Jain \& Feng [22] propose a latent palmprint matching technique consisting of partial to full palmprints for forensic applications, improving the feature extraction algorithm proposed by [20]. Although the appropriateness of this approach, we encounter the problem of large computational demand on Discrete Fourier Transform and Gabor Filtering, and additionally an apparent requirement of about 150 minutiae per partial palmprint. To achieve an acceptable classification rate, a fusion of multiple partial palmprints is needed from the same palmprint. This is an important demand, since sometimes, only one sample is available.

More recent approaches propose different matching criteria than merely minutiae analysis. A document presented by Dai \& Zhou [23] proposes a multi-feature fusion algorithm that, compared to the latent matching elaborated by Jain \& Feng, presents an improved matching percentage of $91.7 \%$, however the method is not presented for partial to full matching. On 2012, Dai et al. [24] propose a robust ridge-based matching algorithm which outperforms the techniques presented in [22] and [23]. However, it is again unclear if the method works for partial palmprints and, since it's based on more features than the minutiae, requires a higher definition and quality than 
images found on, for example, a crime scene. A method based on wavelets has just been presented [25], although the interesting results, this method does not work on partial palmprints due to the need of representing the whole ridges. Nibouche et. al. presented in [26] a method to obtain a fingerprint distance but it needs several samples of the same full palmprint since it is based on PCAs. In [27], PCAs are also used, but each palmprint is divided into several square-overlapping blocks. This was done to classify these blocks into either a good block or a non-palmprint block. Finally, in the paper presented by Wang et. al.[28], palmprint images are decomposed by $2 \mathrm{D}$ Gabor wavelets. The drawback of this methodology is that this decomposition is very sensitive to the length of the obtained palmprint and again, cannot be used to satisfy our requirements.

While most of the palmprint matching approaches are based on a full-to-full association, our contribution is based on a tiny partial section of the palmprint being associated with its complete counterpart (and only one sample is available), which is a more plausible scenario in forensics studies. Only the method presented in [22] considered this fact although they need a larger partial palmprint than our requirements.

\subsection{Definitions}

Following the same nomenclature as section 2, two minutiae $m_{i}^{P}$ and $m_{j}^{F_{a}}$ extracted from palmprints $P$ and $F_{a}$ are represented by positions $\left(x_{i}^{P}, y_{i}^{P}\right)$ and $\left(x_{j}^{F_{a}}, y_{j}^{F_{a}}\right)$ and two features $f_{i}^{P}=\left(\theta_{i}^{P}, t_{i}^{P}\right)$ and $f_{j}^{F a}=\left(\theta_{j}^{F_{a}}, t_{j}^{F a}\right)$. Feature $\theta_{i}$ is the directional angle of the ridge at the minutia point and $t_{i}$ represents the type of minutia (termination or bifurcation) [12].We need to define the distance between features. If $t_{i}^{P}=t_{j}^{F a}$ then both minutiae are terminal or bifurcation, then $\operatorname{dist}_{P, M}^{\text {feature }}\left(f_{i}^{F_{a}}, f_{j}^{P}\right)=$ cyclical_dist $\left(\theta_{i}^{\prime F_{a}}, \theta_{j}^{\prime P}\right)$. Otherwise, these minutiae cannot be mapped since they belong to different types and so $\operatorname{dist}\left(f_{i}^{F_{a}}, f_{j}^{P}\right)=\infty$. Minutiae angles, $\theta_{i}^{\prime P}$ and ${\theta^{\prime}}_{j}^{F_{a}}$ are the original ones that have been normalised depending the average angle. $\theta_{i}^{\prime P}=\theta_{i}^{P}-$ $\bar{\theta}^{P}$ and $\theta_{i}^{\prime F_{a}}=\theta_{i}^{F_{a}}-\bar{\theta}^{F_{a}}$. This is done to be independent of (to the most) the rotation.

The distance between positions is defined as follows, $\operatorname{dist}_{P, M}^{\text {position }}\left(\left(x_{i}^{P}, y_{i}^{P}\right),\left(x_{j}^{F_{a}}, y_{j}^{F_{a}}\right)\right)=$ Euclidean_dist $\left(\left(x_{i}^{\prime P}, y_{i}^{\prime P}\right),\left(x_{j}^{\prime F_{a}}, y_{j}^{\prime F_{a}}\right)\right)$ where $\left(x_{i}^{\prime}, y_{i}^{\prime}\right)$ is the position of minutiae $\left(x_{i}^{P}, y_{i}^{P}\right)$ in which a translation to the centre $(\bar{x}, \bar{y})$ has been applied and also a rotation. The angle of this rotation is the average angle $\bar{\theta}^{P}$ of the minutiae in the partial palmprint. Similarly, $\left(x_{j}^{\prime F_{a}}, y_{j}^{\prime F_{a}}\right)$ is the position of $\left(x_{j}^{F_{a}}, y_{j}^{F_{a}}\right)$ translated to the centre of $F_{a}$. And also a rotation of the mean angle $\bar{\theta}^{F_{a}}$ has been applied. That is, $\left(x_{i}^{\prime}, y_{i}^{\prime P}\right)=\operatorname{rotate}_{\bar{\theta}^{P}}\left(\left(x_{i}^{P}, y_{i}^{P}\right)-\left(x^{C}, y^{C}\right)\right)$ and $\left(x_{j}^{\prime F_{a}}, y_{j}^{\prime F_{a}}\right)=$ rotate $_{\bar{\theta}^{F_{a}}}\left(\left(x_{j}^{F_{a}}, y_{j}^{F_{a}}\right)-\left(x_{a}^{C}, y_{a}^{C}\right)\right)$. 
Having defined the positional and feature distances, we define the distance between two minutiae $m_{i}^{P}$ and $m_{j}^{F_{a}}$ as follows $\operatorname{dist}\left(m_{i}^{P}, m_{j}^{F_{a}}\right)=$

$$
w^{\text {feature }} \cdot \operatorname{dist}_{P, M}^{\text {feature }}\left(f_{i}^{F_{a}}, f_{j}^{P}\right)+w^{\text {position }} \cdot \operatorname{dist}_{P, M}^{\text {position }}\left(\left(x_{i}^{P}, y_{i}^{P}\right),\left(x_{j}^{F a}, y_{j}^{F a}\right)\right)
$$

where weights $w^{\text {feature }}$ and $w^{\text {position }}$ depend on the data.

Finally, given two partial palmprints, the distance between them is defined as follows, $D\left(P, F_{a}\right)=\min _{\forall l_{a}} \frac{\sum_{m_{i}^{P}} \operatorname{dist}\left(m_{i}^{P}, m_{l_{a}(i)}^{F a}\right)}{|P|}$.

\subsection{Algorithm}

To compare the efficiency of our method, the only processes throughout our method that we have to define are the point extractor and the registration process. The other processes are independent of the application. On the one hand, we always used the minutiae extractor defined in [22], [23], [24] to extract the minutiae from each image, both the partial and the full. On the other hand, we used the Hough method proposed in [29] and the ICP method [5] as the registration processes to compare with our own. The first method, although fingerprint oriented, was considered since it is able to work with few minutiae. The second method was selected since it is a general method used to match points. Notice that in this application, we do not need to obtain the transformation matrix $H_{a}$. The matching algorithm only returns the correspondence between minutiae and the final result.

\subsection{Dataset and Experimentation Process}

We have used images contained in the Tsinghua 500 PPI Palmprint Database [20]. It is a public high-resolution palmprint database composed of 500 palmprint images of 2040 × 2040 resolution and captured with a commercial palmprint scanner from Hisign. We selected the first 10 subjects of the database [21]. From each of these subjects, 8 images of his or her palm are enrolled. We then considered the first four palmprints belong to the reference set, and the last four belong to the test set. Therefore, the full palmprints are the same as the reference set, and the partial palmprints are a circular patch (given a variable radius and a random centre) of a palmprint on the test set. We used the algorithm presented in [22], [23], [24] to extract the minutiae from each image, obtaining an average of 800 minutiae per palmprint.

Figure 4 shows on the $y$-axis the recognition ratio of our algorithm ( $P F$ Registration) in comparison to Hough [5] and ICP [29] respect to the radius (in centimetres)of the patch image. We realise that our method performs better than the two other approaches, particularly for tiny images (between 1 and $3 \mathrm{~cm}$ radius). If the radius is bigger than 3 centimetres, both PF-Registration and ICP will deliver similar results, while the Hough method takes bigger radius $(>10 \mathrm{~cm})$ to match the success of our technique. 


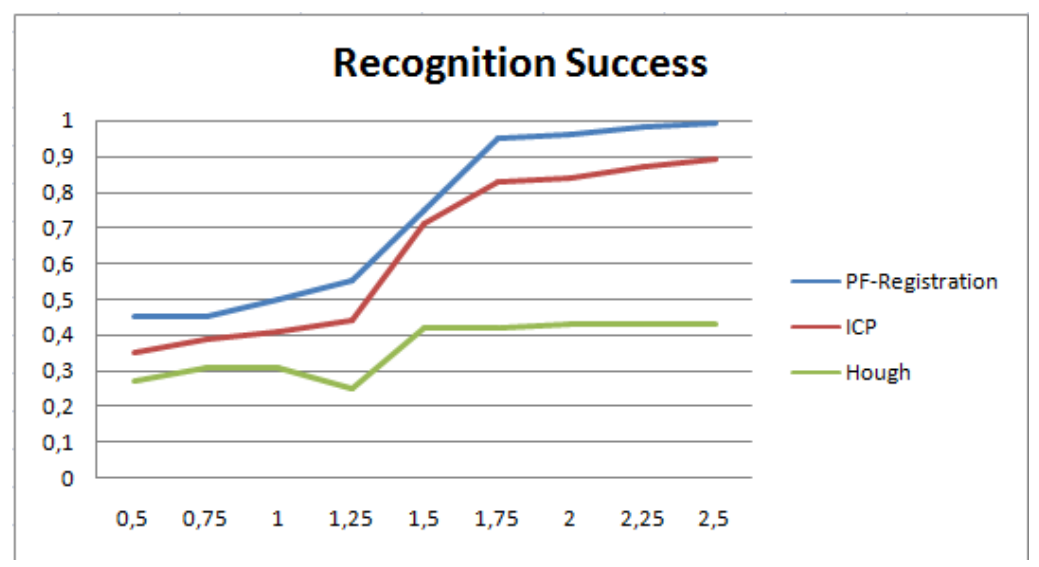

Fig. 4. Recognition ratio respect to the radius in centimetres

\section{Conclusions}

We have presented a method to perform image registration in which one of the images is supposed to be a tiny patch of the other one. This is a current situation in some applications such as object detection in large scenes or palmprint forensic identification. We have used known methods and the new contribution of this paper is simply to put together these methods and define a specific model. Moreover, in the experimental validation we have shown that it is a useful model since we achieve to perform image registration with really small patches.

\section{References}

[1] Salvi, J., Matabosch, C., Fofi, D., Forest, J.: A review of recent range image registration methods with accuracy evaluation. Image Vision Comput. 25(5), 578-596 (2007)

[2] Goshtasby, A.A.: 2-D and 3-D Image Registration for Medical, Remote Sensing, and Industrial Applications. Wiley Press (2005)

[3] Zitová, F.: Image registration methods: a survey. Image Vision Com. 21(11), 977-1000 (2003)

[4] Mikolajczyk, K., Schmid, C.: A performance evaluation of local descriptors. IEEE Trans. Pattern Anal. Mach. Intell. 27(10), 1615-1630 (2005)

[5] Zhang, Z.: Iterative point matching for registration of free-form curves and surfaces. Int. J. Comput. Vision 13(2), 119-152 (1994)

[6] Kuhn, H.W.: The Hungarian method for the assignment problem Export. Naval Research Logistics Quarterly 2(1-2), 83-97 (1955)

[7] Fischler, M.A., Bolles, R.C.: Random sample consensus: a paradigm for model fitting with applications to image analysis and automated cartography. Commun. ACM 24(6), 381-395 (1981)

[8] Sanromà, G., Alquézar, R., Serratosa, F., Herrera, B.: Smooth Point-set Registration using Neighbouring Constraints. Pattern Recognition Letters 33, 2029-2037 (2012) 
[9] Kokiopoulou, E., Frossard, P.: Graph-based classification of multiple observation sets. Pattern Recognition 43, 3988-3997 (2010)

[10] Kokiopoulou, E., Frossard, P.: Graph-based classification of multiple observation sets. Pattern Recognition 43, 3988-3997 (2010)

[11] Wachinger, C., Navab, N.: Simultaneous Registration of Multiple Images: Similarity Metrics and Efficient Optimization. IEEE Trans. on Pattern Analysis and Matching Intelligence 35(5), 1221-1233 (2013)

[12] Jain, A.K., Flynn, P., Ross, A.A.: Handbook of Biometrics. Springer (2009)

[13] Ballard, D.H.: Generalizing the Hough Transform to Detect Arbitrary Shapes", Ridge Based Palmprint Matching. IEEE Trans. on Pattern Analysis and Matching Intelligence (1980)

[14] Kassim, A.A., Tan, T., Tan, K.H.: A comparative study of efficient generalised Hough transform techniques. Image and Vision Computing 17, 737-748 (1999)

[15] Kuhn, H.W.: The Hungarian method for the assignment problem Export. Naval Research Logistics Quarterly 2(1-2), 83-97 (1955)

[16] Yashodha, G., Bremananlh, R.: Rotation Invariant Palmprint Recognition: An Overview an Implementation. In: MVIP (2012)

[17] Somvanshi, P., Rane, M.: Survey of Palmprint Recognition. International Journal of Scientific \& Engineering Research 3(2) (2012)

[18] Zhang, D., Zuo, W., Yue, F.: A Comparative Study of Palmprint Recognition Algorithms. ACM Computing Surveys 44(1), 2 (2012)

[19] Kong, W.K., Zhang, D.: Using Low-Resolution Palmprint Images and Texture Analysis for Personal Identification. In: ICPR (2002)

[20] Funada, J., et al.: Feature Extraction Method for Palmprint Considering Elimination of Creases. In: Proc. 14th Int. Conf. Pattern Recognition, pp. 1849-1854 (1998)

[21] http://deim.urv.cat/ francesc.serratosa/databases /

[22] Jain, A.K., Feng, J.: Latent Palmprint Matching. IEEE Trans. on PAMI (2009)

[23] Dai, J., Zhou, J.: Multifeature-Based High-Resolution Palmprint Recognition. IEEE Trans. Pattern Analysis and Machine Intelligence 33(5), 945-957 (2011)

[24] Dai, J., Feng, J., Zhou, J.: Robust and Efficient Ridge Based Palmprint Matching. IEEE Transactions on Pattern Analysis and Matching Intelligence 34(8) (2012)

[25] Wang, X., Liang, J., Wang, M.: On-line fast palmprint identification based on adaptive lifting wavelet scheme. Knowledge-Based Systems 42, 68-73 (2013)

[26] Nibouche, O., Jiang, J., Trundle, P.: Analysis of performance of palmprint matching with enforced sparsity. Digital Signal Processing 22(2), 348-355 (2012)

[27] Badrinath, G.S., Gupta, P.: Palmprint based recognition system using phase-difference information. Future Generation Computer Systems 28(1), 287-305 (2012)

[28] Wang, X., Lei, L., Wang, M.: Palmprint verification based on 2D - Gabor wavelet and pulse-coupled neural network. Knowledge-Based Systems 27, 451-455 (2012)

[29] Ratha, N.K., Karu, K., Chen, S., Jain, A.K.: A Real-Time Matching System for Large Fingerprint Databases. IEEE Trans. on PAMI 18(8), 799-813 (1996)

[30] Serratosa, F.: Fast Computation of Bipartite Graph Matching. Pattern Recognition Letters, PRL 45, 244-250 (2014) 